\title{
RIPK1 Inhibitor GSK3145095
}

National Cancer Institute

\section{Source}

National Cancer Institute. RIPK1 Inhibitor GSK3145095. NCI Thesaurus. Code C156737.

An orally available, small-molecule inhibitor of receptor-interacting serine/threonineprotein kinase 1 (RIPK1; receptor-interacting protein 1; RIP1) with potential antineoplastic and immunomodulatory activities. Upon administration, GSK3145095 disrupts RIPK1mediated signaling, which may reduce C-X-C motif chemokine lig and 1 (CXCL1)-driven recruitment and migration of immunosuppressive myeloid-derived suppressor cells (MDSCs) in the tumor microenvironment (TME). This allows effector cells, such as natural killer (NK) cells and cytotoxic T-lymphocytes (CT Ls), to kill and eliminate cancer cells. RIPK1, a serine-threonine kinase that normally plays a key role in inflammation and cell death in response to tissue damage and pathogen recognition, is overexpressed in certain cancer types and may be associated with oncogenesis and promotion of the immunosuppressive nature of the TME. 